determinants of SA, though little is known about how siblings influence risk of SA. We assessed whether the risk of SA is associated with sibling's SA. We also explored underlying causes by examining sibling SA associations in sibling pairs of varying degrees of similarity.

Methods The study population was all individuals born in Norway between 1967 and 1976. We identified 215106 employed individuals with at least one sibling. Our study outcome was first SA during 2001-2003. The main study determinant was sibling SA during 2000, and covariates were gender, type of sibship, type of SA, and both siblings' educational level. We examined the association of a sibling having an SA during 2001 with the index person's likelihood of SA during the following 3 years.

Results The 3 -year risk of at least one absence was $31.8 \%$ $(\mathrm{N}=65718)$. The presence of sibling SA conferred a RR for index person's SA of 1.41 for men (95\% CI 1.37 to 1.45$)$ and 1.22 for women (95\% CI 1.19 to 1.25). Higher RRs were seen when siblings were more similar. RRs were higher when siblings had the same educational level than if siblings had different educational level. RRs were higher for twins than full siblings, which again were higher than for half siblings.

Conclusions Risk of SA is associated with sibling SA, and there is a pattern suggesting that the association is stronger when siblings are more similar.

\title{
IS RISK OF SICKNESS ABSENCE ASSOCIATED WITH
} SIBLING SICKNESS ABSENCE?

Karina Corbett, Ingrid Sivesind Mehlum, Odd 0 Aalen, Petter Kristensen NIOH, Os/o, Norway

10.1136/oemed-2011-100382.126

Objectives Sickness absence (SA) is an important measure of work-related health. Early family factors are important 